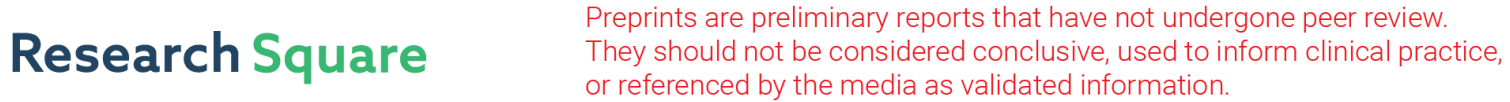 \\ Multi-modality MRI fusion for Alzheimer's disease detection using deep learning
}

\section{Latifa Houria ( $\sim$ latifahouria@gmail.com )}

Universite Saad Dahlab Blida

Noureddine Belkhamsa

Universite Saad Dahlab Blida

\section{Assia Cherfa}

Universite Saad Dahlab Blida

\section{Yazid Cherfa}

Universite Saad Dahlab Blida

\section{Research Article}

Keywords: Diffusion tensor imaging, Structural MRI, Alzheimer's disease, Convolutional Neural Network, Support Vector Machine

Posted Date: February 21st, 2022

DOl: https://doi.org/10.21203/rs.3.rs-1053938/v1

License: (c) (1) This work is licensed under a Creative Commons Attribution 4.0 International License. Read Full License 


\title{
Multi-modality MRI fusion for Alzheimer's disease detection using deep learning
}

\author{
latifa Houria $^{1 *}$, Noureddine Belkhamsa ${ }^{1 \dagger}$, Assia Cherfa $^{1}$ \\ and Yazid Cherfa ${ }^{1 \dagger}$ \\ $1^{*}$ Department of Electronical Engineering, University of Blida 1 , \\ Blida, Algeria. \\ *Corresponding author(s). E-mail(s): latifahouria@gmail.com; \\ Contributing authors: nourbelk@hotmail.com; assia-bz@yahoo.fr; \\ cherfa-yazid@yahoo.fr; \\ $\dagger$ These authors contributed equally to this work.
}

\begin{abstract}
Diffusion tensor imaging (DTI) is a new technology in magnetic resonance imaging, which allows us to observe the insightful structure of the human body in vivo and non-invasively. It identifies the microstructure of white matter (WM) connectivity by estimating the movement of water molecules at each voxel. This makes possible the identification of the damage to WM integrity caused by Alzheimer's disease $(\mathrm{AD})$ at its early stage, called mild cognitive impairment (MCI). Furthermore, the brain's gray matter (GM) atrophy characterizes the main structural changes in $\mathrm{AD}$, which can be sensitively detected by structural MRI (sMRI) modality. In this research, we further develop a novel multi-modality MRI (DTI and sMRI) fusion strategy to detect WM alterations and GM atrophy in AD patients. The latter is based on a 2dimensional deep convolutional neural network (CNN) features extractor and a Support Vector Machine (SVM) classifier. The fusion framework consists of merging features extracted from DTI scalar metrics (fractional anisotropy (FA) and mean diffusivity (MD)), and GM using 2D-CNN and feeding them to SVM to classify AD vs. cognitively normal (CN), $\mathrm{AD}$ vs. MCI, and MCI vs. CN. Our novel multimodal AD method demonstrates a superior performance with an accuracy of $99.79 \%$, $99.6 \%$, and $97.00 \%$ for $\mathrm{AD} / \mathrm{CN}, \mathrm{AD} / \mathrm{MCI}$, and $\mathrm{MCI} / \mathrm{CN}$ respectively.
\end{abstract}

Keywords: Diffusion tensor imaging, Structural MRI, Alzheimer's disease, Convolutional Neural Network, Support Vector Machine 


\section{Introduction}

Alzheimer's disease (AD) is an irreversible progressive neurodegenerative disorder that affects people over the age of 65 and outlines around $60 \%$ of dementia worldwide. It is caused by damage to nerve cells in certain brain regions, affecting a persons memory and cognitive abilities, which disrupt their daily life. The Alzheimer's Association declares that AD is the sixth leading cause of death in the USA; around 50 million people were diagnosed with this disease in 2018 and in 2050, this number will be tripled (1). At present, no effective treatment or prevention is found. Moreover, disease management is prohibitively costly. Early screening of this disease is of primordial importance for researchers to slow down its progression and optimize the treatment. In this context, advances in neuroimaging, primarily magnetic resonance imaging (MRI), have shown the potential to improve the early diagnosis of AD.

$\mathrm{AD}$ is characterized by a progressive loss of Gray matter (GM) that occurs pre-symptomatically in certain neuro-anatomical structures (2). Structural MRI (sMRI) is the most used neuroimaging modality to detect brain atrophy. It has already highlighted many biomarkers of Alzheimer's disease; in particular, the atrophy of structures such as the hippocampus, the amygdala, and the thalamus (3). In fact, hippocampal atrophy in prodromal patients proved to be the best structural predictor of Alzheimer's disease progression (4). However, it is associated with a large number of neurodegenerative pathologies, thereby limiting its specificity to Alzheimer's disease (5).

Within this frame of reference, many studies on the AD-prodromal phase called mild cognitive impairment (MCI) have focused their research on the hippocampus. Nevertheless, some other structures appear interesting such as, the volume of the amygdala which could be a structural predictor as powerful or even more efficient than the volume of the hippocampus to predict MCI $(6 ; 7)$. Furthermore, there are changes in white matter that preceded gray matter atrophy but are not detectable by sMRI (8). The introduction of diffusion tensor imaging allows identification of these changes when the patient still presents an MCI (9). The MCI is the transitory phase between (CN) decline and $\mathrm{AD}$ or another dementia.

DTI has conventionally studied the white matter microstructural integrity based on the estimation of the water molecules' diffusion in all directions (six directions at least) (10). The degree of anisotropy of water diffusion is represented by the fractional anisotropy (FA), while mean diffusivity (MD) represents its magnitude. Studies have shown the importance of measuring these two DTI indices (FA and MD) to describe the physiological aging in the MCI patient phase (11). Increased MD and decreased FA were reported in $\mathrm{AD}$ patients compared to CN. Higher MD in MCI patients was observed in both hippocampi (12). Indeed, a considerable increase in MD and decrease in FA indicates a progressive loss of the barriers restricting the motion of water 
molecules in tissue compartments, associated with neuronal loss in AD (13). It, therefore, seems important to measure the DTI indices because they can provide additional information on the pathophysiology of the disease.

The introduction of machine learning and deep learning techniques has greatly contributed to the diagnosis and prognosis of $\mathrm{AD}$ based on neuroimaging data (14). Numerous research works have been published for the AD classification using DTI, where the FA and MD were the most frequently metrics used as features. The most popular among these machine learningbased methods utilized as classifiers, are the Support Vector Machine (SVM), and Random Forest $(\mathrm{RF})(15 ; 16 ; 17 ; 18 ; 19)$. Most of them used the tractbased spatial statistics (TBSS) algorithm (37) to extract the white matter skeleton from FA and MD. They selected only the pertinence WM Skeleton information to perform binary or multi-classification using Alzheimers disease national initiative (ADNI) data set. The difference was presented in the classification task, where Maggipinto (18) used Random Forest and Lella (19) proposed to concatenate the best result from different classifiers (SVM, $\mathrm{RF}$, and Multi-layer perceptron (MLP)) from all features groups (FA, MD, radial diffusivity (RD), longitudinal diffusivity (LD)). The use of DTI-based machine learning shows impressive performance. However, it is necessary to extract features and subsequently select the relevant ones to perform classification tasks, which is difficult and time-consuming.

Deep learning is a state-of-the-art machine learning method (20). Classification techniques using deep convolutional neural networks (CNN) revealed higher AD detection performance (21). Most of the literature approaches have used CNN-based sMRI to classify the different stages of Alzheimer's disease. CNN can handle low to high automatic feature extraction from complex structures. Some authors have proposed a new CNN architecture (22) reaching promising results with an accuracy of $99.9 \%$. Others have reported excellent results using transfer learning methods (23; 24; 25). However, others have suggested extracting deep discriminative features based on transfer learning methods and classifying them with SVM $(26 ; 27)$.

In recent years, DTI indices, principally MD, combined with sMRI information have been adopted by many researchers. They proposed different techniques to combine DTI and sMRI. Massalimova et al. (28) have tried multi-modal Resnet-18 network (sMRI and DTI) in classifying CN, MCI, and AD from OASIS-3 datasets. They managed to suggest that the classification performed by the softmax layer could be preferable than another classifier in contrast to Kang et al. (26). Kang et al. (26) suggested a fusion technique consisting of merging slices with the same index of the T1w, FA, and MD images into an RGB slice. After that, the pre-trained VGG16 network is used to extract the features and SVM classifier to discriminate MCI patients, from CN using the ADNI dataset. Aderghal et al. (29) proposed LeNet-like CNN based 
on sMRI and DTI-MD images. They selected the median slice Hippocampal and its two neighbors in each projection (axial, sagittal, and coronal). The proposed CNN is trained on the MNIST database. They first retrained the model on sMRI then on DTI-MD. They achieved a classification accuracy of $86.83 \%$ for $\mathrm{AD}$ vs. CN, $69.85 \%$ for MCI vs. CN, and $71.75 \%$ for $\mathrm{AD}$ vs. MCI. Marzban et al. (30) proposed a simple 2DCNN based on a single convolution layer. They trained the model on diffusion scalars metrics (FA, MO, and MD) and GM. The cascaded MD and GM volumes achieved an overall accuracy of $88.9 \%$ and $79.6 \%$ respectively for $\mathrm{AD}$ vs. CN and MCI vs. CN. Ahmed et al. (31) extracted visual features from the hippocampus ROI in both sMRI and MD images. The extracted features and the amount of CSF calculated on the sMRI are combined and classified using multi-kernel learning (MKL).

Assessment of pathophysiological changes by neuroimaging would be essential to predict AD. Single modality cannot provide enough information, therefore, multi-modality must be combined to detect AD. sMRI and DTI have received more attention in recent years to study the progression of Alzheimer's disease. These two modalities are complementary; the sMRI detect the shrinkage of gray matter and changes in the brain volume. Moreover, the DTI is a useful prediction marker to detect the WM deterioration. In this context, we aim to detect patterns of micro and macrostructural changes in the different AD stages using the multi-modality MRI (sMRI and DTI) fusion process. We propose a new methodology that consists of a new CNN to extract the salient visual features from the DTI measurements and the GM images separately. After that, these features are merged and transmitted to SVM to identify AD from MCI, AD from CN, and MCI from CN.

\section{Database}

Dataset used in this work has been obtained from the Alzheimer's Disease Neuroimaging Initiative (ADNI) (http://adni.loni.usc.edu). The ADNI was launched in 2003 as a public-private partnership, led by Principal Investigator Michael W. Weiner, MD. The objectives of the ADNI study are the identification of biomarkers for clinical use and early detection of $\mathrm{AD}$ (32). The selected balanced dataset includes both Diffusion-weighted images (DWI) and sMRI brain scans from 150 individuals of both genders $(50 \mathrm{AD}, 50 \mathrm{CN}$, and $50 \mathrm{MCI}$ ), with ages varying from 55 to 90 acquired by GE medical system scanners. The 50 MCI subjects are selected with 25 early MCI and 25 Late MCI. The selected subjects coming from ADNI-GO and ADNI-2 phases.

The raw 3D T1-weighted sMRI were acquired with $256 \times 256 \times 196$ voxels per volume, a voxel size of $1.0 \times 1.0 \times 1.2 \mathrm{~mm} 3$, inversion time $=400 \mathrm{~ms}$, and flip angle $=11$. The raw DWI data was acquired with $128 \times 128$ matrix, a voxel size of $2.7 \times 2.7 \times 2.7 \mathrm{~mm}^{3}$, and 41 gradient directions $\left(b=1000 \mathrm{~s} / \mathrm{mm}^{2}\right)$. 
In addition to these images, 5 T2-weighted images without diffusion $(b=0)$ are used as reference scans. More informtion about the acquisition parameters can be found in the ADNI2 protocol.

\section{Methodology}

Our proposed strategy consists of pre-processing, a 2D slice selection, a features extraction, and a classification. We work on DTI measurements (FA, MD) and GM brain segmented from T1-weighted sMRI to classify (CN vs. AD), ( $\mathrm{AD}$ vs. $\mathrm{MCI}$ ), and (CN vs. MCI). New 2DCNN architecture was trained by slice-level dataset (only the 32 relevant slices selected from FA, MD, GM images) to extract the salient features from DTI maps and GM. The optimal FA-CNN, MD-CNN, and GM-CNN models are saved depending on lower loss value during the training process, then adapted to extract features from the last fully connected layer. After that, the features of each slice in the subjectlevel dataset (FA, MD, GM) are extracted by their optimal model (FA-CNN, MD-CNN, and GM-CNN). These features are merging and feeding to the SVM classifier to improve the performance as is illustrated in figure 1 . The detailed description is found in the following subsections.

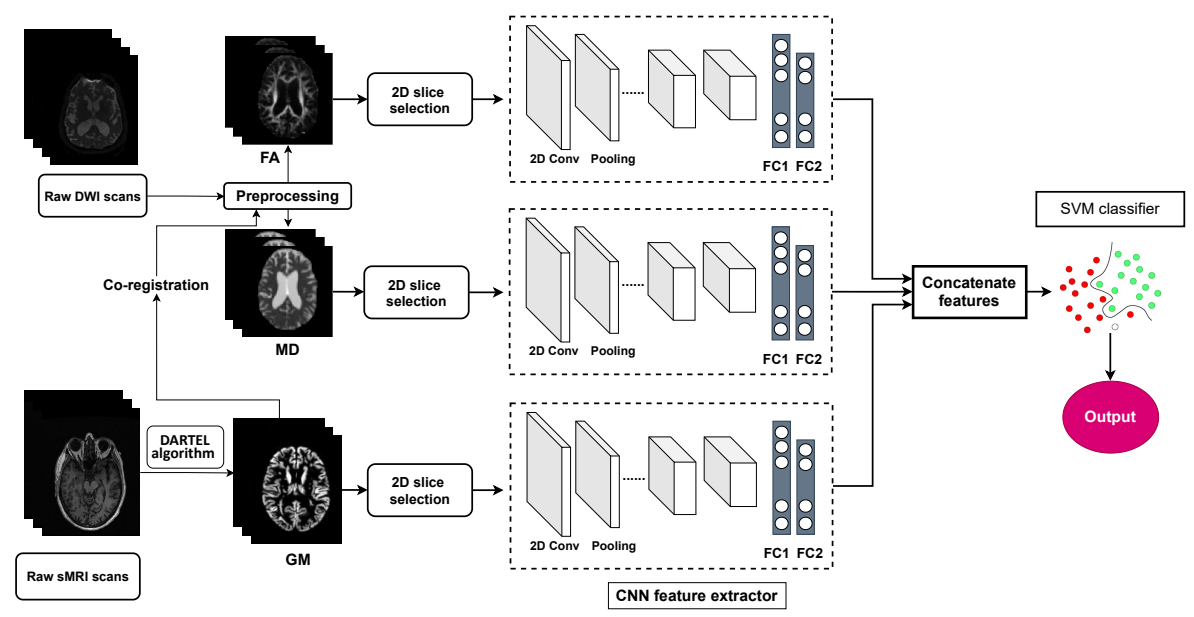

Fig. 1 Flowchart of the proposed fusion multi-modalities system using the 2DCNN-SVM approach for AD identification.

\subsection{Pre-Processing}

All downloaded data are converted from DICOM format to Neuroimaging InFormatics Technology Initiative (NIFTI) format, using MRICro software (http://www.mricro.com/). 
The pre-processing steps of the raw sMRI volumes to segment the GM are performed by the CAT12 toolbox (http://www.neuro.uni-jena. de/cat/). The CAT12 toolbox is an extension of SPM12 software (33) . In short, all T1weighted 3D sMRI are normalized by the DARTEL algorithm (Diffeomorphic Anatomic Registration Through Exponentiated Lie algebra) using an affine transformation followed by a nonlinear registration, corrected for bias field inhomogeneities, and then segmented into GM, WM components.

DWI volumes are preprocessed using Functional Magnetic Resonance Imaging of the Brain (FMRIB) Software Library (FSL) (34). First DWI scans are corrected for eddy current distortions and susceptibility artefacts by the FSL-eddy correct. FSLs Brain Extraction Tool was used to remove the brain skull. The diffusion tensor calculations are performed by the FSL dtifit at each voxel of fixed DWI scans. The eigenvalues of the diffusion tensor $(\lambda 1, \lambda 2$ $, \lambda 3)$ were utilized to obtain maps of scalar anisotropy and diffusivity. Several diffusion metrics can be calculated. The widely used diffusion metrics are fractional anisotropy (FA) and mean diffusivity (MD). FA is calculated using equation 1. MD represents the magnitude of diffusion which is calculated by averaging the three eigenvalues as it is mentioned in equation 2. Finally, FA and MD are co-registered with the corresponding sMRI scans and each scan contains $121 \times 145 \times 121$ voxels using SPM12.

$$
\begin{gathered}
F A=\sqrt{\frac{1}{2}} \frac{\sqrt{\left(\lambda_{1}^{2}-\lambda_{2}^{2}\right)+\left(\lambda_{2}^{2}-\lambda_{3}^{2}\right)\left(\lambda_{3}^{2}-\lambda_{1}^{2}\right)}}{\lambda_{1}^{2}+\lambda_{2}^{2}+\lambda_{3}^{2}} \\
M D=\frac{\lambda_{1}+\lambda_{2}+\lambda_{3}}{3}
\end{gathered}
$$

\section{$3.22 \mathrm{D}$ slice selection}

Each FA, MD, and GM volume is decomposed into 2D slices along the axial view to highlight the most distinctive features and ensure improved classification efficiency. We select 32 slices from each subject based on higher entropy information (slices with indices 3465). The selected slices are associated with most of the deteriorated $\mathrm{AD}$ brain regions mentioned in literature such as the hippocampus, the entorhinal cortex, and thalamus. As a result, a total of $1600(32 \times 50)$ of each class (CN, MCI, and AD) are selected. More details are shown in table1.

Table 1 Sample size of the preprocessed selection process

\begin{tabular}{lll}
\hline Classes & Number of patients & Number of slices \\
\hline AD & 50 & 1600 \\
CN & 50 & 1600 \\
MCI & 50 & 1600 \\
\hline
\end{tabular}




\subsection{Feature extraction using $2 \mathrm{DCNN}$}

The handcrafted features extraction was the main problem in the traditional machine learning algorithms which is hard and time-consuming. CNN can perform this task automatically without human intervention.

CNN is the most common deep learning model used among neural networks. It is inspired by the human visual system. A typical CNN architecture comprises principally an input layer, convolution layer, pooling layer, fully connected layer, and classification layer. The convolution layer extracts automatically the features from the input FA, MD, or GM images by multiplying element-wise with a filter. The pooling layer aimed to reduce the redundant information by acquiring the average of a region or the maximum. The fully connected layer is used to reduce and transform the feature maps to a column feature map. The classifiers are finally used for $\mathrm{AD}$ prediction.

In short, the 2DCNN architecture consists of three convolutional layers with $3 \times 3$ size filters. Each convolutional layer is followed by a RELU layer, batch normalization (BN) layers, and a max-pooling layer, then two fully connected layers, softmax layer, and output layer. The RELU layer sets the negative values to zero and BN accelerates the training process. More details are tabulated in table 2 .

Table 2 Layers Proprieties for the proposed 2DCNN architecture.

\begin{tabular}{|c|c|c|}
\hline Layers (\#) & Layer Name & Layer Properties \\
\hline 1 & Input layer & $145 \times 121 \times 1$ images \\
\hline 2 & Convolutional & $323 \times 3 \times 1$ convolutions with stride $\left[\begin{array}{ll}1 & 1\end{array}\right]$ and padding same \\
\hline 3 & Batch Normalization & 32 \\
\hline 4 & Rectified Linear Unit & Rectified Linear Unit \\
\hline 5 & Max pooling & $2 \times 2$ max pooling with stride $\left[\begin{array}{ll}2 & 2\end{array}\right]$ and padding $\left[\begin{array}{llll}0 & 0 & 0 & 0\end{array}\right]$ \\
\hline 6 & Convolutional & $163 \times 3 \times 32$ convolutions with stride $\left[\begin{array}{ll}1 & 1\end{array}\right]$ and padding same \\
\hline 7 & Batch Normalization & 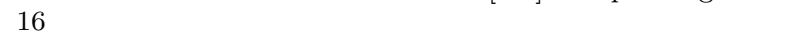 \\
\hline 8 & Rectified Linear Unit & Rectified Linear Unit \\
\hline 9 & Max pooling & $2 \times 2$ max pooling with stride $\left[\begin{array}{ll}2 & 2\end{array}\right]$ and padding $\left[\begin{array}{llll}0 & 0 & 0 & 0\end{array}\right]$ \\
\hline 10 & Convolutional & $83 \times 3 \times 16$ convolutions with stride $\left[\begin{array}{ll}1 & 1\end{array}\right]$ and padding same \\
\hline 11 & Batch Normalization & 8 \\
\hline 12 & Rectified Linear Unit & Rectified Linear Unit \\
\hline 13 & Fully Connected & 256 hidden neurons in fully connected layer \\
\hline 14 & Fully Connected & 2 hidden neurons in fully connected layer \\
\hline 15 & Softmax & Softmax \\
\hline 16 & Classification Output & 2 output classes \\
\hline
\end{tabular}

\subsection{Classification using support vector machine (SVM)}

SVM is a widely applied supervised learning method that treats small highdimensional data by finding a maximal margin hyperplane to separate classes 
and solve a binary classification problem (35). SVM is considered better to use than the Softmax layer as is mentioned in previously published studies $(36 ; 37)$. The trained FA-CNN, MD-CNN, and GM-CNN are adopted to extract the features. These features are then transmitted to the SVM classifier instead of the Softmax layer for AD classification. These features extracted from FA, MD, and GM images is a matrix whose size is the number of slices multiplied by the number of features selected from each slice. For 32 slices of each subject, the feature representation has the dimension of $32 \times 2$. For all subjects (100), the output of each model is a matrix of $10032 \times 2$. They are then concatenated into a total feature matrix with the dimension of $3200 \times 2$. SVM classifier is trained and tested using these deep extracted features as is shown in figure 2.

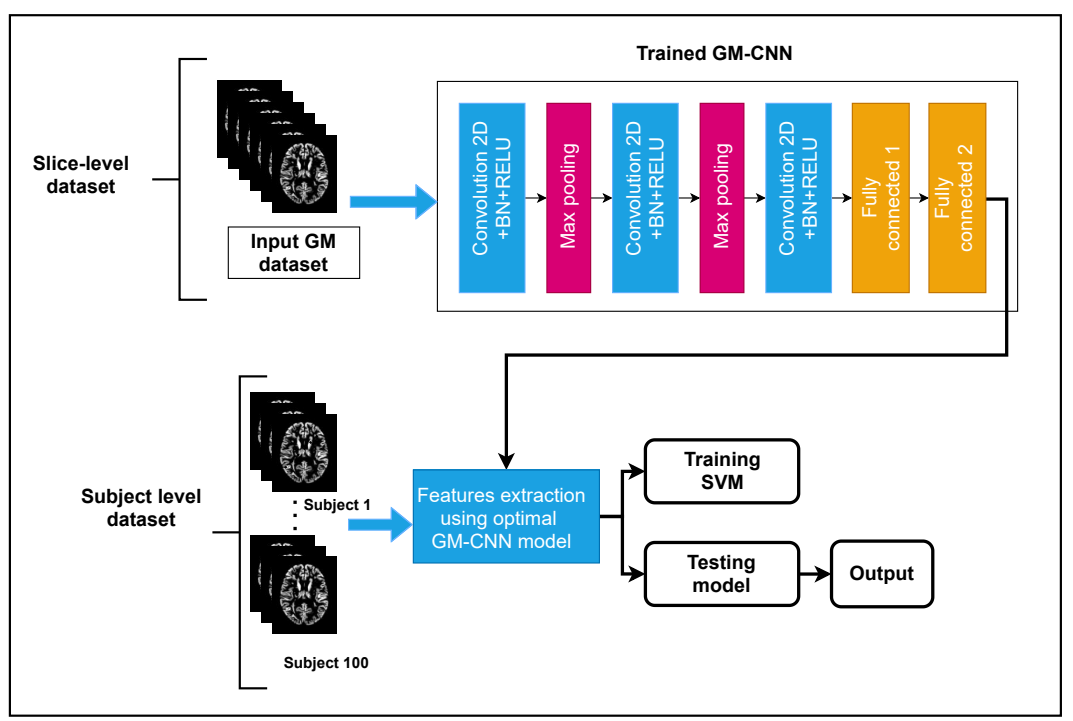

Fig. 2 The pipeline of proposed GM-CNN with SVM method to distinguish between AD and $\mathrm{CN}$.

\subsection{Multi-modality MRI fusion process.}

The automatic AD screening fusion algorithm developed using multimodalities MRI is illustrated in figure 1. The three optimal CNN (FA-CNN, MD-CNN and, GM-CNN) are used to extract features. We tried several fusion procedures experiences (FA and MD), (FA and GM), (MD and GM), and (FA and $\mathrm{MD}$ and GM) to choose the best model score. The fusion process consists of merging the features extracted from FA, MD, and GM into a global feature vector. Accordingly, the size of the fused FA + MD + GM feature matrix is $3200 \times 6$. 


\section{Results}

\subsection{Experiments}

In this work, several experiments are carried out to validate the effectiveness of our proposed method to classify (AD vs. $\mathrm{CN}$ ), (CN vs. MCI), and (AD vs. MCI). In the first experience, we performed a direct unimodal classification of features extracted from FA, MD, and GM. This gives us information about the best modality and map. In the second experiment, we study whether multimodality increases performance and allows better discrimination between the different classes or not. This is achieved by studying the impact of merging features of the two modalities.

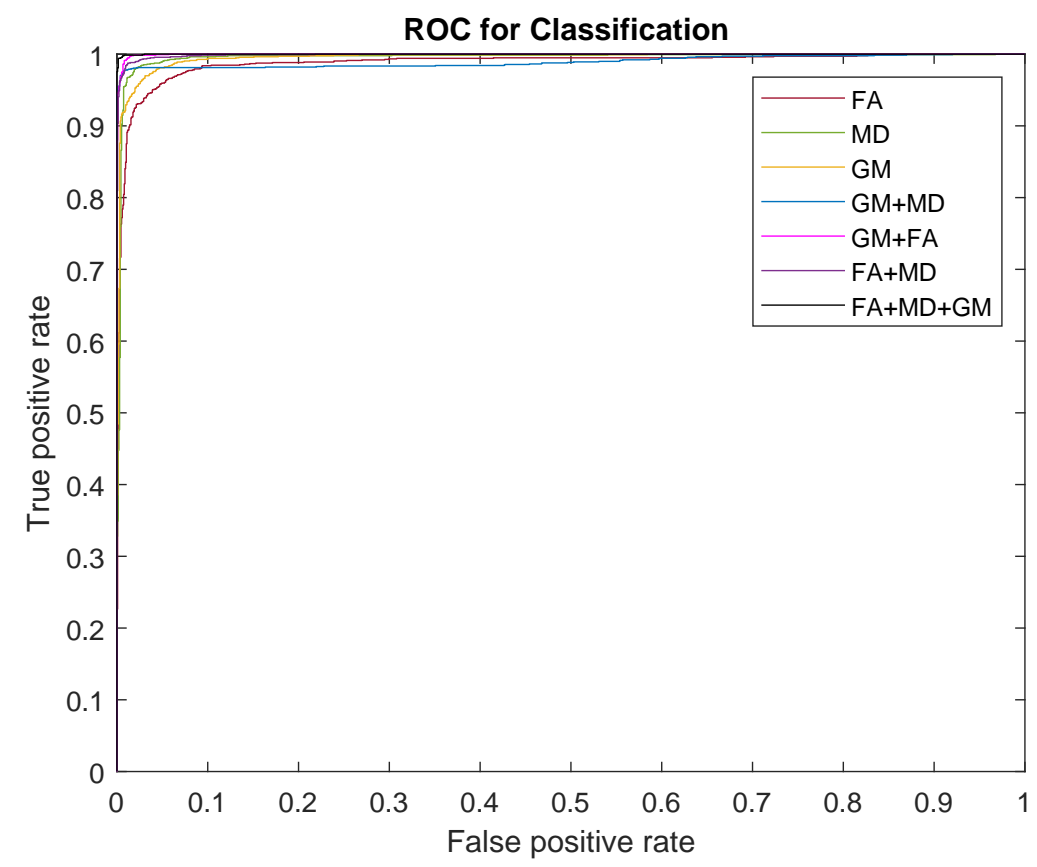

Fig. 3 ROC curves of $\mathrm{AD}$ vs. $\mathrm{CN}$

The 2DCNN-SVM proposed has been implemented using MATLAB ver. R2019a and running on a $3.1 \mathrm{GHz}$ Intel-i7 processor, $16 \mathrm{~GB}$ of RAM. The CNN model was trained using an optimized SGDM (Stochastic Gradient Descent Momentum) using the back-propagation algorithm and cross-entropy as a loss function. The batch size is 64 , the learning rate is 0.0001 for 25 epochs. There is a total of 3200 images of each map (FA, MD, and GM), 1600 images for each class. The dataset is divided into $75 \%$ for training, $15 \%$ for validation and the remaining $15 \%$ for testing the SVM. The same CNN 


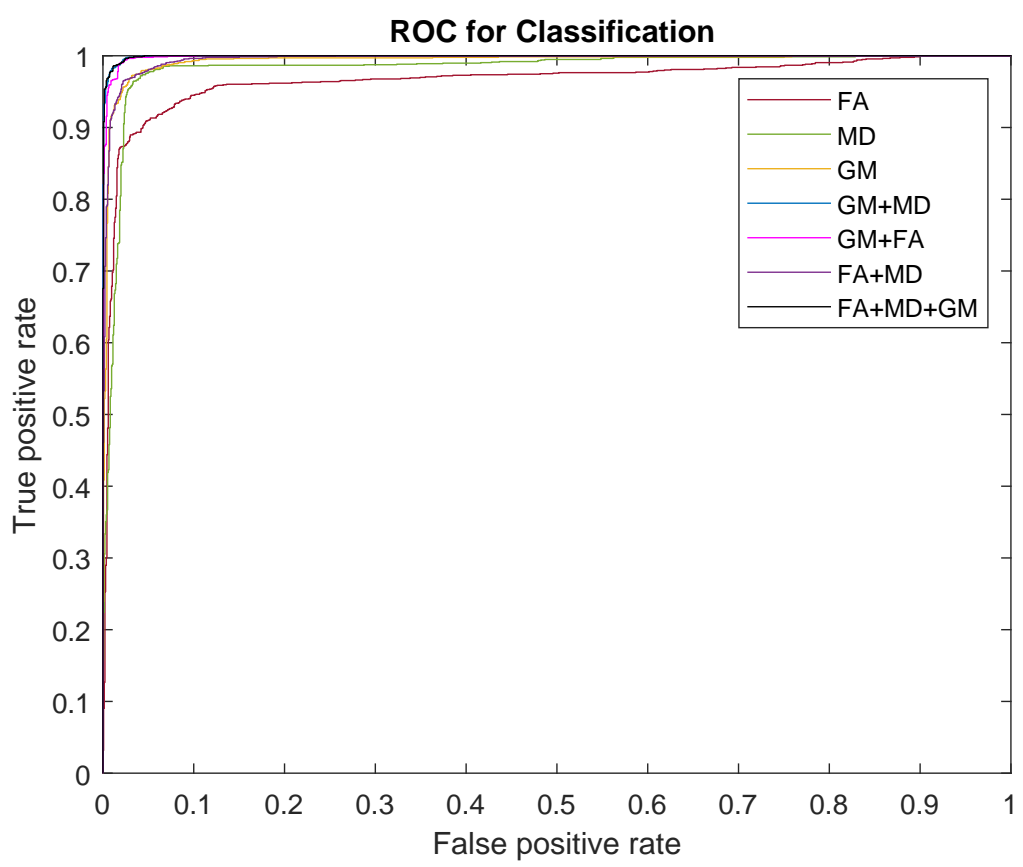

Fig. 4 ROC curves of $\mathrm{AD}$ vs. MCI.

architecture is used to train FA slices, MD slices and GM slices. For the SVM classifier, the extracted data is categorized into training, validation, and test data. We used the extracted features from 2720 images for the training and 480 images for the test.

The best SVM using radial basis function (RBF) (Gaussian kernel) classification score was obtained by 10 -fold cross-validation. The optimal hyperparameters (cost and gamma) were determined using the grid search technique. It finds the best model result from different combinations of parameters; where cost controls the error and gamma gives the curvature weight of the decision boundary.

\subsection{Evaluation}

The performance of our method was validated using accuracy and the area under the receiver operating characteristic curve (AUC). The validation results are illustrated in table 3 and the ROC curves of 10-fold cross-validation are shown in figures $3,4,5$.

The fused FA, MD, and GM improved better the result and outperformed the single modality and the sMRI+MD fused procedures adopted in many previous studies $(26 ; 29 ; 30)$. 


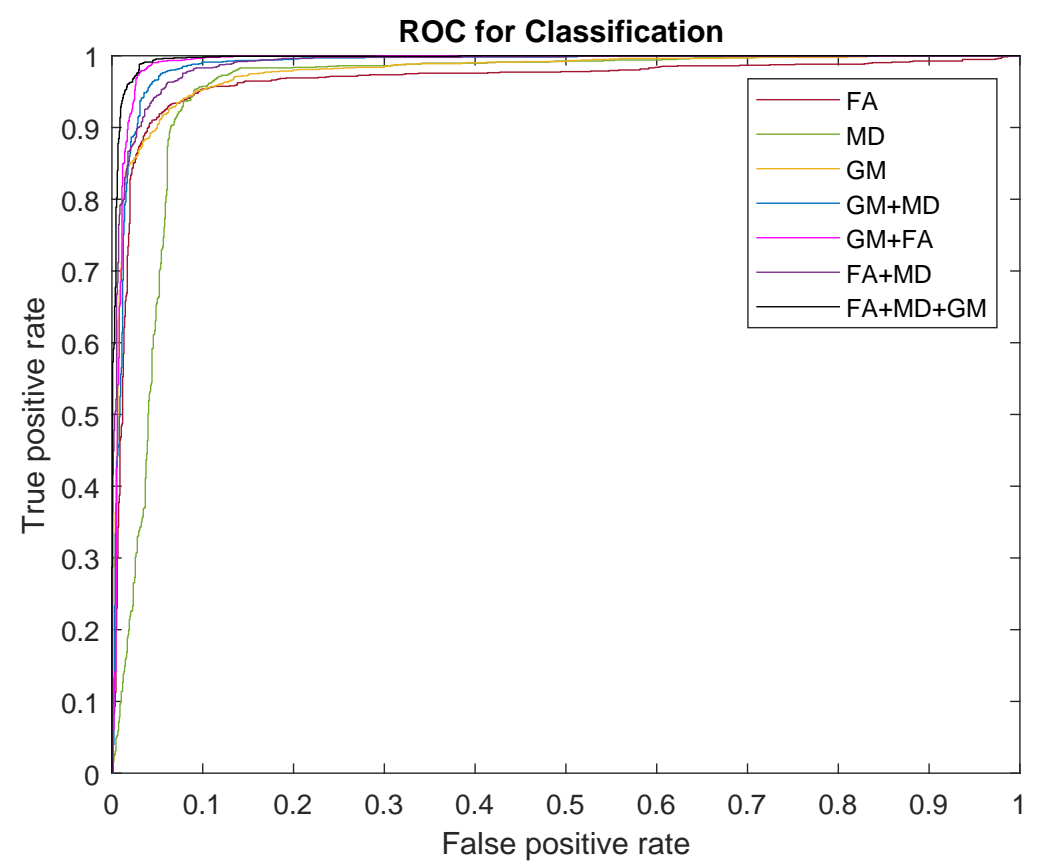

Fig. 5 ROC curves of CN vs. MCI.

Table 3 The performance of the validation dataset.

\begin{tabular}{|c|c|c|c|c|c|c|c|c|}
\hline \multirow{2}{*}{ Classes } & \multirow{2}{*}{$\begin{array}{l}\text { Modality } \\
\text { Map }\end{array}$} & \multicolumn{3}{|c|}{ DTI } & \multirow{2}{*}{$\begin{array}{l}\text { sMRI } \\
\text { GM }\end{array}$} & \multicolumn{3}{|c|}{ sMRI+DTI } \\
\hline & & FA & $\mathrm{MD}$ & $\mathrm{FA}+\mathrm{MD}$ & & $\mathrm{GM}+\mathrm{FA}$ & GM+MD & $\begin{array}{l}\text { GM+FA } \\
+\mathrm{MD}\end{array}$ \\
\hline \multirow{2}{*}{$\mathrm{AD} / \mathrm{CN}$} & Acc & 0.9548 & 0.9794 & 0.9779 & 0.9691 & 0.9912 & 0.9849 & 0.9952 \\
\hline & AUC & 0.9879 & 0.9952 & 0.9891 & 0.9951 & 0.9996 & 0.9897 & 0.9999 \\
\hline \multirow{2}{*}{$\mathrm{AD} / \mathrm{MCI}$} & Acc & 0.9298 & 0.9621 & 0.9695 & 0.9687 & 0.9849 & 0.9868 & 0.9908 \\
\hline & AUC & 9642 & 0.9902 & 0.9954 & 0.9931 & 0.9998 & 0.9993 & 0.9996 \\
\hline \multirow{2}{*}{ CN/MCI } & Acc & 0.9331 & 0.9276 & 0.9485 & 0.9303 & 0.9717 & 0,9599 & 0.9779 \\
\hline & AUC & 0.9643 & 0.9518 & 0.9883 & 0.9772 & 0.9906 & 0.9865 & 0.996 \\
\hline
\end{tabular}

We tested our method using $240 \mathrm{AD}$ images, $240 \mathrm{CN}$ images, and 240 MCI images. The used evaluation metrics are the accuracy, sensitivity, and specificity determined by the confusion matrices. In matrices of confusion, the sensitivity is shown in the last row and the specificity in the last column. The diagonal boxes indicate the numbers and percentages of correctly classified classes and the last one shows the overall accuracy of the model. An example of the confusion matrix of the fused characteristics FA, MD, and GM is shown in figure $6,7,8$. All test results are summarized in Table 4. 


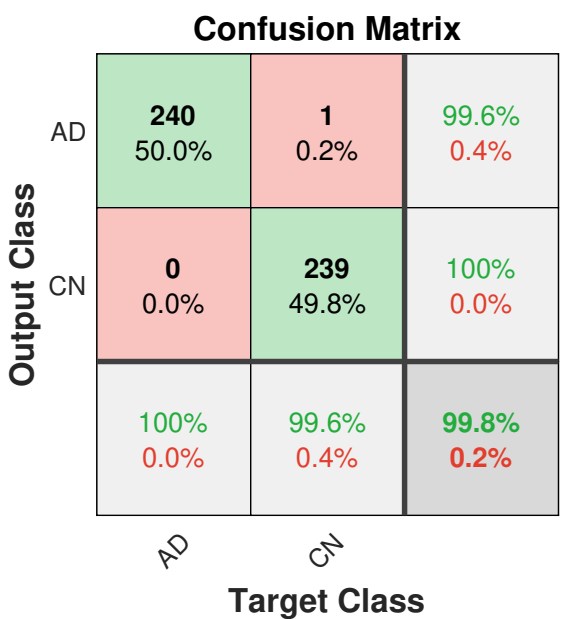

Fig. 6 Matrice of confusion of $\mathrm{AD}$ vs. CN.

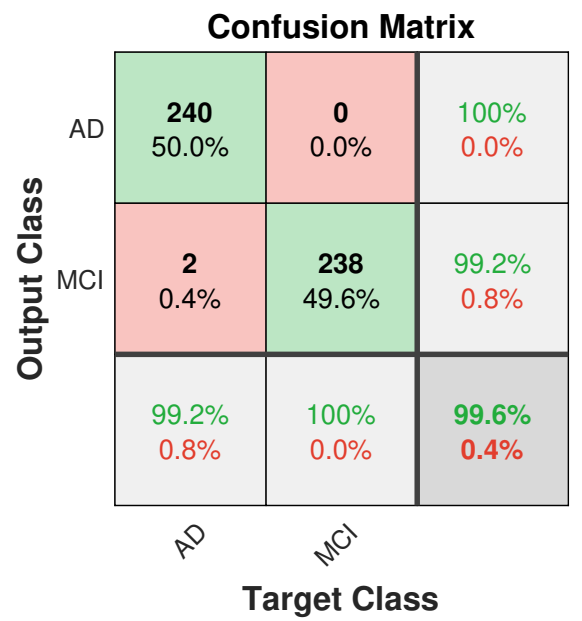

Fig. 7 Matrice of confusion of AD vs. MCI.

Table 4 shows that the FA, MD, and GM are important to discriminate the different $\mathrm{AD}$ stages. For the use of FA, MD, GM independently, we report that MD obtained the best result in the case of AD vs. CN with an accuracy of $98.96 \%$. However, the GM yields better results in classifying AD vs. MCI and CN vs. MCI with an accuracy of $96.88 \%$ and $93.50 \%$ respectively.

We investigated the best combination of features (FA and MD, FA and GM, and MD and GM). Fused FA and MD outperformed the other combined features with an accuracy of $99.98 \%$ and $98.33 \%$ to classify AD vs. CN and $\mathrm{AD}$ vs. MCI. On the other hand, fused GM and MD achieved higher results to classify CN vs. MCI with an accuracy of $97.00 \%$, a sensitivity of $97.20 \%$, and a 


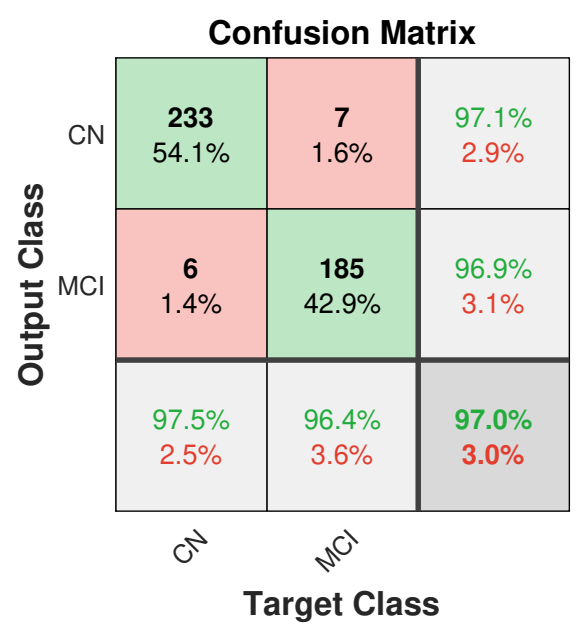

Fig. 8 Matrice of confusion of $\mathrm{CN}$ vs. MCI.

Table 4 Performance evaluation of the proposed 2DCNN-SVM technique on the test dataset.

\begin{tabular}{|c|c|c|c|c|c|c|c|c|}
\hline \multirow{2}{*}{ Classes } & \multirow{2}{*}{$\begin{array}{l}\text { Modality } \\
\text { Map }\end{array}$} & \multicolumn{3}{|c|}{ DTI } & \multirow{2}{*}{$\frac{\text { sMRI }}{\mathrm{GM}}$} & \multicolumn{3}{|c|}{ sMRI+DTI } \\
\hline & & FA & $\mathrm{MD}$ & $\mathrm{FA}+\mathrm{MD}$ & & $\mathrm{GM}+\mathrm{FA}$ & $\mathrm{GM}+\mathrm{MD}$ & $\begin{array}{l}\mathrm{GM}+\mathrm{F} \\
\sim \\
+\mathrm{MD}\end{array}$ \\
\hline \multirow{4}{*}{$\mathrm{AD} / \mathrm{CN}$} & Acc & 0.9750 & 0.9896 & 0.9989 & 0.9625 & 0.9850 & 0.9938 & 0.9979 \\
\hline & Sen & 0.9750 & 0.9900 & 0.9875 & 0.9625 & 0.9855 & 0.9940 & 0.9980 \\
\hline & Spec & 0.9750 & 0.9900 & 0.9875 & 0.9625 & 0.9850 & 0.9940 & 0.9980 \\
\hline & Acc & 0.9375 & 0.9542 & 0.9833 & 0.9688 & 0.9896 & 0.9813 & 0.9958 \\
\hline \multirow{3}{*}{$\mathrm{AD} / \mathrm{MCI}$} & Sen & 0.9400 & 0.9545 & 0.9835 & 0.9690 & 0.9900 & 0.9820 & 0.9960 \\
\hline & Spe & 0.9375 & 0.9545 & 0.9835 & 0.9685 & 0.9895 & 0.9815 & 0.9960 \\
\hline & Acc & 0.9230 & 0.9210 & 0,9440 & 0.9350 & 0.9560 & 0.9700 & 0.9700 \\
\hline \multirow[t]{2}{*}{ CN/MCI } & Sen & 0.9225 & 0.9220 & 0.9450 & 0.9360 & 0.9590 & 0.9720 & 0.9695 \\
\hline & Spec & 0.9220 & 0.9180 & 0.9420 & 0.9325 & 0.9520 & 0.9680 & 0.9700 \\
\hline
\end{tabular}

specificity of $96.80 \%$. Compared to the use of the single modality, the merged FA, MD, and GM led to an increase of approximately $3.54 \%, 5.83 \%, 4.1 \%$ for the accuracy, $3.55 \%, 5,85 \%, 4.75 \%$ for the sensitivity, and $2.8 \%, 5.85 \%, 5.2 \%$ for the specificity in the three cases of classification (AD vs. CN), (AD vs. $\mathrm{MCI}$ ), and (CN vs. MCI) respectively. So, the multi-modality gives the best performance as clearly shown in figure ??.

\section{Discussion}

To validate the performance and efficiency of our novel workflow, we compared it to the previous approaches presented in the literature and dealing with the same databases (ADNI) and the same modalities (sMRI and DTI). Our results gained higher accuracy in the $\mathrm{AD}$ detection compared to other 
Table 5 Comparison of results with state-of-the-art techniques applied to AD detection.

\begin{tabular}{|c|c|c|c|c|c|c|c|c|c|}
\hline \multirow{2}{*}{ Study } & \multirow{2}{*}{ Modality } & \multicolumn{3}{|c|}{ Subject size } & \multirow{2}{*}{$\begin{array}{c}\text { Method } \\
\text { (Maps and classifier) }\end{array}$} & \multicolumn{4}{|c|}{ Classification accuracy (\%) } \\
\hline & & $\mathrm{AD}$ & $\mathrm{CN}$ & MCI & & $\mathrm{AD} / \mathrm{CN}$ & CN / MCI & MCI/ & $\mathrm{AD}_{\sharp}$ \\
\hline $\begin{array}{l}\text { Lella et al } 2017 . \\
(17)\end{array}$ & DTI & 40 & 40 & - & $\begin{array}{l}\text { (FA, MD), RF, } \\
\text { SVM and } \\
\text { naive Bayes }\end{array}$ & 78.00 & - & - & 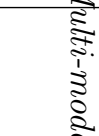 \\
\hline $\begin{array}{l}\text { Maggipinto et al. } \\
2017(20)\end{array}$ & DTI & 89 & 90 & 90 & (FA MD), RF & 87.00 & 81.00 & - & हैं \\
\hline $\begin{array}{l}\text { Ahmed et al. } \\
2017(31)\end{array}$ & DTI+sMRI & 45 & 52 & 58 & $\begin{array}{l}\text { Hippocampus ROI } \\
\text { (sMRI+MD)+CSF } \\
\text { volume, SVM }\end{array}$ & 90.20 & 79.42 & 76.63 & 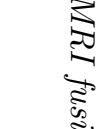 \\
\hline $\begin{array}{l}\text { Aderghal et al. } \\
2020(29)\end{array}$ & DTI+sMRI & 64 & 399 & 273 & $\begin{array}{l}\text { Hippocampus ROI } \\
\text { (sMRI+MD), } \\
\text { LeNet-CNN }\end{array}$ & 92.30 & 78.48 & 79.16 & $\frac{\sqrt{1}}{9}$ \\
\hline $\begin{array}{l}\text { Jiang et al. } \\
2020(27)\end{array}$ & sMRI & - & 50 & 70 & VGG16 with SVM & - & 89.40 & - & $\begin{array}{l}\overrightarrow{8} \\
8 \\
0\end{array}$ \\
\hline $\begin{array}{l}\text { Marzban et al. } \\
2020(30)\end{array}$ & DTI+sMRI & 115 & 185 & 106 & $\begin{array}{l}(\mathrm{MD}, \mathrm{FA}, \mathrm{MO}, \mathrm{GM}) \\
2 \mathrm{DCNN}\end{array}$ & 93.50 & - & 79.6 & 离 \\
\hline $\begin{array}{l}\text { Kang et al. } \\
2020(26)\end{array}$ & DTI+sMRI & - & 50 & 70 & $\begin{array}{c}(\mathrm{FA}+\mathrm{MD}+\mathrm{sMRI}) \\
\mathrm{VGG} 16+\mathrm{SVM}\end{array}$ & - & 94.20 & - & . \\
\hline $\begin{array}{l}\text { Feng et al. } \\
2020(37)\end{array}$ & sMRI & 130 & 135 & 133 & $3 \mathrm{D}-\mathrm{CNN}+\mathrm{SVM}$ & 99.10 & 98.90 & 89.40 & $\begin{array}{l}8 \\
8 \\
5 \\
5 \\
5\end{array}$ \\
\hline $\begin{array}{l}\text { Lella et al. } \\
2021 \text { (19) }\end{array}$ & DTI & 40 & 40 & - & $\begin{array}{l}(\mathrm{FA}+\mathrm{MD}+\mathrm{RD}+\mathrm{LD}), \\
\mathrm{SVM}, \mathrm{RF}, \mathrm{PML}\end{array}$ & 88.50 & - & - & $\frac{8}{8}$ \\
\hline $\begin{array}{l}\text { Naz et al. } \\
2021(24)\end{array}$ & sMRI & 95 & 146 & 138 & VGG16, VGG19 & 98.89 & 97.06 & 99.27 & 胥. \\
\hline Our approach & DTI+sMRI & 50 & 50 & 50 & $\begin{array}{l}(\mathrm{FA}+\mathrm{MD},+\mathrm{GM}), \\
2 \mathrm{DCNN}-\mathrm{SVM}\end{array}$ & 99.79 & 99.58 & 97.00 & \\
\hline
\end{tabular}




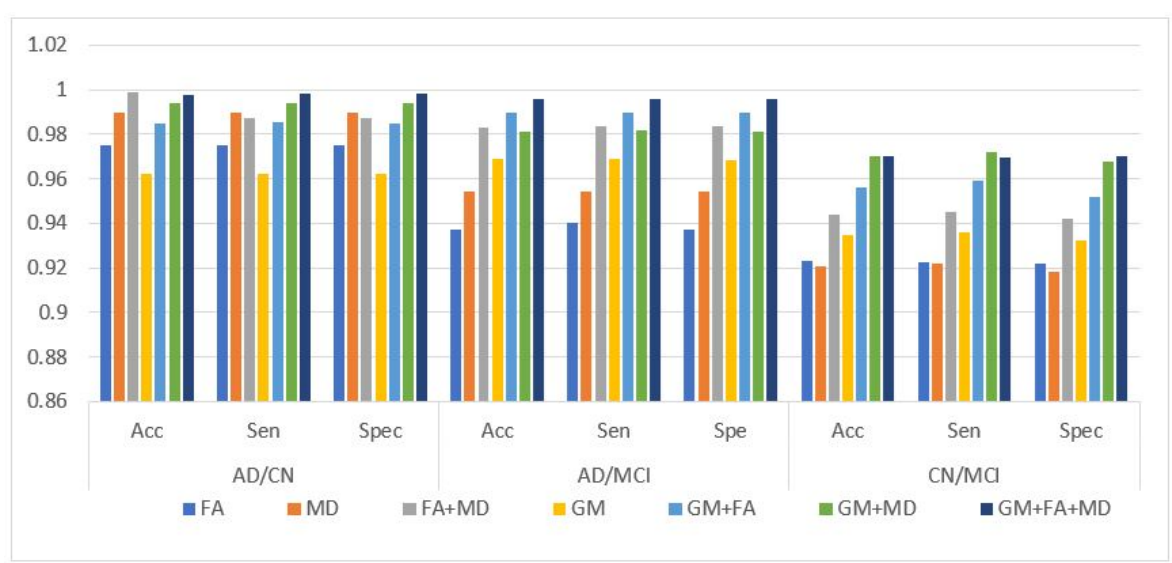

Fig. 9 Comparison of performance of proposed technique for binary classification of AD vs. $\mathrm{CN} A D$ vs. MCI, and CN vs. MCI.

studies as is shown in Table 5 .

In general, our results concerning $\mathrm{AD}$ early detection imply the existence of distinct pathophysiological processes. In fact, the hippocampus is known to be one of the earliest and most severely damaged structures affected by $\mathrm{AD}$. However, there are other structures involved in AD detection such as the amygdala, thalamus, and putamen. The relevant slices selection seems a powerful and easy method than segmenting the hippocampus or other brains regions which requires a human expert. Our network learns the complex patterns of brain atrophy from relevant sections that contain almost all of the AD-affected regions mentioned in the literature, for each patient. This eliminates the process of segmentation of the hippocampus and other regions of the brain. Moreover, a subsequent selection of the most discriminating characteristics is avoided in our approach.

Our results confirm the effectiveness of the DTI measurement FA and MD in the classification of $\mathrm{AD}$ vs. $\mathrm{CN}, \mathrm{AD}$ vs. $\mathrm{MCI}$, and $\mathrm{CN}$ vs. MCI which is consistent with the previous works $(19 ; 18)$. In addition, The GM atrophy in sMRI is of great interest to researchers for the AD early detection. The sMRI based transfer learning has proven impressive results $(23 ; 25)$. Generally, the VGG16 and VGG19 models have gained higher accuracy than other pretrained models (24). Recently, some of the authors(26; 27) succeeded in using a pre-trained (VGG16) model for automatic extraction of features and SVM for the classification; they achieved a higher accuracy. However, the transfer learning technique relies generally on natural images whose models are trained using the Imagenet database (39). Conversely, our simple networks learn and extract from scratch the most pertinent features. 
In the past few years, the multi modalities (DTI-MD and sMRI) were reported by many researchers. They proposed different combination techniques to ensure the best classification. Aderghal et al. (29) suggested the transfer learning technique to perform the fusion process and Marzban et al. (30)adopted a cascaded CNN. However, they achieved lower accuracy than what we got which is over $97 \%$. This is probably due to the small sample size we used compared to them, or the fact that we didn't work on specific ROI, or the impact of adding FA.

In summary, both, diffusion scales metrics and the GM are powerful elements and important for AD stage discrimination. The multi-modality fusion process $(\mathrm{FA}+\mathrm{MD}+\mathrm{GM})$ seems to be the best technique to improve the $\mathrm{AD}$ classification performance.

\section{Conclusion}

In this paper, we have proposed a 2DCNN-SVM classification approach based on DTI scalar metrics (FA and MD) and GM segmented from T1w images from ADNI databases for $\mathrm{AD}$ detection and diagnosis. The fusion of features extracted from FA, MD, and GM by the proposed 2DCNN demonstrates the effectiveness of our method achieving a classification accuracy of $99.79 \%$, $99.85 \%$, and $97.00 \%$ for $\mathrm{AD} / \mathrm{CN}, \mathrm{AD} / \mathrm{MCI}$, and CN/MCI respectively. In conclusion; the use of DTI-FA, DTI-MD, and GM separately gives lower results than fused together.

Acknowledgments. This project was funded by the LASICOM laboratory of University of Blida 1, Department of Electrical Engineering. The authors would like to acknowledge the Alzheimers Disease Neuroimaging Initiative (ADNI) for the public sharing of these precious neuroimaging data (http://adni.loni.usc.edu). We are thankful to the General Directorate of Scientific Research and Technological Development (DGRSDT) for their support in developing this work.

\section{Declarations}

- Funding The authors declare that they have no conflict of interest.; it is an academic PhD study.

- Conflict of interest/Competing interests No conflict of interest.

- Ethics approval This article does not contain any studies with human participants or animals performed by any of the authors.

- Availability of data and materials The data used in this study were obtained from the Alzheimers Disease Neuroimaging Initiative (ADNI). The full data can be downloaded from https:(http://adni.loni.usc.edu).

- Code availability The code that supported the fndings of this study is available on request from the corresponding author (Latifa Houria). 
- Authors' contributions All authors were involved in the work leading up to the manuscript. All sources used are properly disclosed (correct citation).

\section{References}

[1] 2018. CP (2018.) The state of the art of dementia research: New frontiers. World Alzheimers Report 2018 (2018).

[2] Fox N, Warrington E, Freeborough P, Hartikainen P, Kennedy A, Stevens J, Rossor MNJB (1996) Presymptomatic hippocampal atrophy in Alzheimer's disease: A longitudinal MRI study. 119 (6):2001-2007

[3] Pini L, Pievani M, Bocchetta M, Altomare D, Bosco P, Cavedo E, Galluzzi S, Marizzoni M, Frisoni GBJArr (2016) Brain atrophy in Alzheimers disease and aging. 30:25-48

[4] Apostolova LG, Dinov ID, Dutton RA, Hayashi KM, Toga AW, Cummings JL, Thompson PMJB (2006) 3D comparison of hippocampal atrophy in amnestic mild cognitive impairment and Alzheimer's disease. 129 (11):2867-2873

[5] Van de Pol L, Gertz H-J, Scheltens P, Wolf HJND (2011) Hippocampal atrophy in subcortical vascular dementia. 8 (6):465-469

[6] Bell-McGinty S, Lopez OL, Meltzer CC, Scanlon JM, Whyte EM, Dekosky ST, Becker JTJAon (2005) Differential cortical atrophy in subgroups of mild cognitive impairment. 62 (9):1393-1397

[7] Klein-Koerkamp Y, A Heckemann R, T Ramdeen K, Moreaud O, Keignart S, Krainik A, Hammers A, Baciu M, Hot P, Research AsdNIJCA (2014) Amygdalar atrophy in early Alzheimers disease. 11 (3):239-252

[8] Ringman JM, ONeill J, Geschwind D, Medina L, Apostolova LG, Rodriguez Y, Schaffer B, Varpetian A, Tseng B, Ortiz FJB (2007) Diffusion tensor imaging in preclinical and presymptomatic carriers of familial Alzheimer's disease mutations. 130 (7):1767-1776

[9] Medina D, deToledo-Morrell L, Urresta F, Gabrieli JD, Moseley M, Fleischman D, Bennett DA, Leurgans S, Turner DA, Stebbins GTJNoa (2006) White matter changes in mild cognitive impairment and AD: A diffusion tensor imaging study. 27 (5):663-672

[10] Basser PJ, Mattiello J, LeBihan DJBj (1994) MR diffusion tensor spectroscopy and imaging. 66 (1):259-267

[11] Cherubini A, Pran P, Caltagirone C, Sabatini U, Spalletta GJN (2009) Aging of subcortical nuclei: microstructural, mineralization and atrophy 
modifications measured in vivo using MRI. 48 (1):29-36

[12] Fellgiebel A, Wille P, Mller MJ, Winterer G, Scheurich A, Vucurevic G, Schmidt LG, Stoeter PJD, disorders gc (2004) Ultrastructural hippocampal and white matter alterations in mild cognitive impairment: a diffusion tensor imaging study. 18 (1):101-108

[13] Kantarci K, Avula R, Senjem M, Samikoglu A, Zhang B, Weigand S, Przybelski S, Edmonson H, Vemuri P, Knopman DSJN (2010) Dementia with Lewy bodies and Alzheimer disease: neurodegenerative patterns characterized by DTI. 74 (22):1814-1821

[14] Billeci L, Badolato A, Bachi L, Tonacci AJP (2020) Machine Learning for the Classification of Alzheimers Disease and Its Prodromal Stage Using Brain Diffusion Tensor Imaging Data: A Systematic Review. 8 (9):1071

[15] Dyrba M, Ewers M, Wegrzyn M, Kilimann I, Plant C, Oswald A, Meindl T, Pievani M, Bokde AL, Fellgiebel AJPo (2013) Robust automated detection of microstructural white matter degeneration in Alzheimers disease using machine learning classification of multicenter DTI data. 8 (5):e64925

[16] O'Dwyer L, Lamberton F, Bokde AL, Ewers M, Faluyi YO, Tanner C, Mazoyer B, O'Neill D, Bartley M, Collins DRJPo (2012) Using support vector machines with multiple indices of diffusion for automated classification of mild cognitive impairment. 7 (2):e32441

[17] Lella E, Amoroso N, Bellotti R, Diacono D, La Rocca M, Maggipinto T, Monaco A, Tangaro S Machine learning for the assessment of Alzheimer's disease through DTI. In: Applications of Digital Image Processing XL, 2017. International Society for Optics and Photonics, p 1039619

[18] Maggipinto T, Bellotti R, Amoroso N, Diacono D, Donvito G, Lella E, Monaco A, Scelsi MA, Tangaro S, Medicine AsDNIJPi, Biology (2017) DTI measurements for Alzheimers classification. 62 (6):2361

[19] Lella E, Pazienza A, Lof D, Anglani R, Vitulano FJE (2021) An Ensemble Learning Approach Based on Diffusion Tensor Imaging Measures for Alzheimers Disease Classification. 10 (3):249

[20] Lee G, Fujita H (2020) Deep learning in medical image analysis: challenges and applications, vol 1213. Springer,

[21] Hazarika RA, Abraham A, Sur SN, Maji AK, Kandar DJIJoMIR (2021) Different techniques for Alzheimers disease classification using brain images: a study.1-20 
[22] Kundaram SS, Pathak KC Deep Learning-Based Alzheimer Disease Detection. In: Proceedings of the Fourth International Conference on Microelectronics, Computing and Communication Systems. Springer, pp $587-597$

[23] Ashraf A, Naz S, Shirazi SH, Razzak I, Parsad MJMT, Applications (2021) Deep transfer learning for alzheimer neurological disorder detection.1-26

[24] Naz S, Ashraf A, Zaib AJMS (2021) Transfer learning using freeze features for Alzheimer neurological disorder detection using ADNI dataset.1-10

[25] Maqsood M, Nazir F, Khan U, Aadil F, Jamal H, Mehmood I, Song O-yJS (2019) Transfer learning assisted classification and detection of Alzheimers disease stages using 3D MRI scans. 19 (11):2645

[26] Kang L, Jiang J, Huang J, Zhang TJFian (2020) Identifying early mild cognitive impairment by multi-modality mri-based deep learning. 12:206

[27] Jiang J, Kang L, Huang J, Zhang TJNl (2020) Deep learning based mild cognitive impairment diagnosis using structure MR images. 730:134971

[28] Massalimova A, Varol HAJapa (2021) Input Agnostic Deep Learning for Alzheimer's Disease Classification Using Multimodal MRI Images.

[29] Aderghal K, Afdel K, Benois-Pineau J, Catheline G, Heliyon AsDNIJ (2020) Improving Alzheimer's stage categorization with Convolutional Neural Network using transfer learning and different magnetic resonance imaging modalities. 6 (12):e05652

[30] Marzban EN, Eldeib AM, Yassine IA, Kadah YM, one AsDNIJP (2020) Alzheimers disease diagnosis from diffusion tensor images using convolutional neural networks. 15 (3):e0230409

[31] Ahmed OB, Benois-Pineau J, Allard M, Catheline G, Amar CB, Neurocomputing AsDNIJ (2017) Recognition of Alzheimer's disease and Mild Cognitive Impairment with multimodal image-derived biomarkers and Multiple Kernel Learning. 220:98-110

[32] Petersen RC, Aisen P, Beckett LA, Donohue M, Gamst A, Harvey DJ, Jack C, Jagust W, Shaw L, Toga AJN (2010) Alzheimer's disease neuroimaging initiative (ADNI): clinical characterization. 74 (3):201-209

[33] Penny WD, Friston KJ, Ashburner JT, Kiebel SJ, Nichols TE (2011) Statistical parametric mapping: the analysis of functional brain images. Elsevier, 
[34] Jenkinson M, Beckmann CF, Behrens TE, Woolrich MW, Smith SMJN (2012) Fsl. 62 (2):782-790

[35] Cusano C, Ciocca G, Schettini R Image annotation using SVM. In: Internet imaging $\mathrm{V}, 2003$. International society for optics and photonics, pp 330-338

[36] Raju M, Gopi VP, Anitha V, Wahid KAJP, Medicine ESi (2020) Multiclass diagnosis of Alzheimers disease using cascaded three dimensionalconvolutional neural network. 43 (4):1219-1228

[37] Feng W, Halm-Lutterodt NV, Tang H, Mecum A, Mesregah MK, Ma Y, Li H, Zhang F, Wu Z, Yao EJIJoNS (2020) Automated MRI-based deep learning model for detection of Alzheimers disease process. 30 (06):2050032

[38] Tang X, Holland D, Dale AM, Younes L, Miller MI, mapping AsDNIJHb (2014) Shape abnormalities of subcortical and ventricular structures in mild cognitive impairment and Alzheimer's disease: detecting, quantifying, and predicting. 35 (8):3701-3725

[39] Krizhevsky A, Sutskever I, Hinton GEJAinips (2012) Imagenet classification with deep convolutional neural networks. 25:1097-1105 